\title{
Optimizing Vancomycin Dosing in Obese and Morbidly Obese Patients with MRSA Infections
}

\author{
Eddie Grace*, Nancy Hope Goodbar and Jaime A. Foushee \\ Department of Pharmacy Practice, Presbyterian College School of Pharmacy, 307 North Broad Street, Clinton, USA
}

\begin{abstract}
Approximately 275,000 of these hospitalizations were due to Methicillin-resistant Staphylococcus aureus (MRSA) infections which resulted in over 19,000 deaths. Since VCN's introduction over 6 decades ago, it has been studied in various patient populations such as those with renal disease, burns, and cystic fibrosis. However, until recently, extremely limited data has been available for the use of VCN in obese patients. Due to the differences in VCN pharmacokinetics in obese patients compared to non-obese patients, use of PK parameters in obese patients which were originally derived from non-obese patients results in erroneous dosing of VCN leading to clinical failures and increased incidence of adverse effects. PK parameters such as vancomycin clearance, volume of distribution, protein binding, and creatinine clearance $(\mathrm{CrCl})$ in obese patients will be discussed in addition to studied methods to optimize vancomycin dosing in obese patients by using loading doses, weight based dosing, and area under the curve to minimum inhibitor concentration ratio (AUC:MIC). Due to the variability in dosing of obese patients compared to non-obese patients, VCN maximum doses and the incidence of nephrotoxicity will also be discussed.
\end{abstract}

Keywords: Obesity; Vancomycin; Pharmacokinetics; Mrsa; Loading dose; Nephrotoxicity

\section{Introduction}

In the United States (U.S.), greater than one-third of the adult population over the age of twenty is classified as obese. Obesity accounts for $20 \%$ of individuals under the age of $20 \%$ and accounts for $40 \%$ of adults over the age of sixty, thus signaling an increase in rates of obesity with increased age [1]. In 1990, all states within the U.S. had a prevalence of obesity of less than $15 \%$. Ten years later, the incidence of obesity had increased to nearly $25 \%$ in some states. In 2010 , obesity accounted for up to $34 \%$ of the population in each state. World-wide increases in rates of obesity have resulted in increased incidence of heart diseases, type 2 diabetes mellitus, and certain cancers [1]. The rising rates of these comorbidities such increases healthcare utilization, as these patients are more susceptible to infections requiring hospitalization and intravenous antibiotics. The Centers for Disease Control and Prevention (CDC) estimated that the medical cost related to obesity is approximately 147 billion U.S. dollars annually.

In 2005 , over 475,000 hospitalizations in the U.S. were attributed to Staphylococcus aureus infections [2]. Approximately 275,000 of these hospitalizations were due to Methicillin-resistant Staphylococcus aureus (MRSA) infections which resulted in over 19,000 deaths [2]. [http://www.cdc.gov/mrsa/statistics/index.html]. Due to the rising rate of MRSA infections in both the community and healthcare settings, the use of antibiotics active against MRSA also has been on the rise. Among MRSA active antibiotics, vancomycin (VCN) is often considered the antibiotic of choice for patients hospitalized with suspected or documented MRSA infections. Since the introduction of VCN over 6 decades ago, it has been studied in various patient populations including renal disease, burns, and cystic fibrosis. Until recently, extremely limited data has been available regarding the use of VCN in obese patients. Use of PK parameters originally derived from nonobese patient in an obese population may result in erroneous dosing of $\mathrm{VCN}$, due to the differences in VCN PKs in obese patients compared to non-obese patients. This may result in increased rates of clinical failures and an increased incidence of VCN adverse effects and resistance. This article will discuss PK parameters in obese patients, including
VCN clearance $(\mathrm{VCl})$, volume of distribution $(\mathrm{Vd})$, protein binding, and creatinine clearance $(\mathrm{CrCl})$, as well as methods to optimize VCN dosing in this patient population. Due to the variability in dosing of obese patients compared to non-obese patients, VCN maximum doses and the incidence of nephrotoxicity will also be discussed.

\section{Altered Pharmacokinetics of Vancomycin in Obese Patients}

VCN PKs which have been studied in obese patients including altered Vd due to excess adipose tissue and protein binding. VCN renal elimination has also been studies as it relates to $\mathrm{CrCl}$ and $\mathrm{VCl}$ [3-5]. Based basic PK principles, both $\mathrm{Vd}$ and $\mathrm{VCl}$ play a role in the elimination constant $(\mathrm{Ke})$ which directly relates the dose (D) and dosing interval (T) [6].

The volume of distribution of a drug affects both the loading dose needed to achieve a therapeutic drug level, and the maintenance dose required to maintain a target drug concentration in serum following the loading dose [6]. The variability in VCN Vd variability estimate in previously published studies is likely due to the varying hypotheses surrounding estimates of water content in adipose tissue. The most agreed on estimate is based on aminoglycoside studies in obese patients, which estimated that water content accounts for approximately $30-40 \%$ of adipose tissue weight [7]. Studies in non-obese and obese patients have provided a wide range of values for VCN Vd per kg ranging from

*Corresponding author: Eddie Grace, Pharm.D, BCPS, AQ-ID, AAHIVE, Associate Professor, Department of Pharmacy Practice, Presbyterian College School of Pharmacy, 307 North Broad Street, Clinton, South Carolina 29325, USA, E-mail: EEGrace@presby.edu

Received October 01, 2012; Accepted October 20, 2012; Published October 22 2012

Citation: Grace E, Goodbar NH, Foushee JA (2012) Optimizing Vancomycin Dosing in Obese and Morbidly Obese Patients with MRSA Infections. Adv Pharmacoepidem Drug Safety S1:003. doi:10.4172/2167-1052.S1-003

Copyright: $\odot 2012$ Grace E, et al. This is an open-access article distributed under the terms of the Creative Commons Attribution License, which permits unrestricted use, distribution, and reproduction in any medium, provided the original author and source are credited. 
$0.49 \mathrm{~L} / \mathrm{Kg}$ to $1.25 \mathrm{~L} / \mathrm{Kg}$ based on total body weight (TBW), with specific estimates in obese and morbidly obese patients ranging from $0.26 \mathrm{~L} / \mathrm{Kg}$ (TBW) to as high as $1.7 \mathrm{~L} / \mathrm{Kg}$ (TBW) [8-10]. In addition, some studies suggest that TBW best correlates with Vd while others have shown that replacing TBW with IBW or lean body weight (LBW) does not improve the relationship of weight to VCN Vd $[4,10]$. Utilizing an average Vd of $0.7 \mathrm{~L} / \mathrm{Kg}$ can result in an underestimation of the total Vd if IBW is used. Perhaps similar to aminoglycosides, VCN Vd can be best estimated using an adjusted body weight $(\mathrm{ABW})$ or by use of a lower $\mathrm{Vd} / \mathrm{Kg}$ while continuing to utilize TBW; however, more studies are needed to determine if the use of $\mathrm{ABW}$ is appropriate. $\mathrm{VCl}$ has shown to be increased in obese patients due several physiological changes compared to non-obese patients [3]. $\mathrm{VCl}$ has shown to be approximately 2.5 fold higher in obese patients when compared to non-obese patients $[9,11]$. Using the Cockcroft-Gault equation to estimate $\mathrm{CrCl}$ (which is the standard method used to estimate drug elimination) TBW best correlated with $\mathrm{CrCl}$ and total $\mathrm{VCl}[3,11-14]$.

In addition to altered VCN Vd in obese patients, certain proteins such as a1-acid glycoprotein (AAG) may be elevated in obese patients resulting in an increased amount of VCN bound to serum proteins and lower than expected concentration of unbound (free) VCN in serum [15]. Other studies have shown that other proteins which bind VCN in serum, such as albumin, may be elevated in obese patients compared to non-obese patients [16]. Based on the increased Vd and VCl, an increase in protein binding would result in significant decreases in unbound VCN serum concentration in obese patients leading to a significant increase in clinical failures despite small variations in total VCN concentrations compared to non-obese patients [17].

\section{Altered Vancomycin Pharmacodynamics and Dosing in Obesity}

\section{Vancomycin AUC:MIC ratio and weight based dosing for obesity}

With the PK and pharmacodynamic (PD) changes of VCN seen in the obese population, as well as the dependence of the areas under the concentration curve (AUC) calculation $\mathrm{CrCl}, \mathrm{VCl}$, and $\mathrm{Vd}$, it is evident that body weight is a common denominator in all of these factors. In one study which evaluated initial VCN pharmacokinetic studies in obese patients, TBW had the strongest correlation with $\mathrm{Vd}$ and $\mathrm{VCl}$ compared to IBW and ABW [11]. No significant differences in weight-based daily doses $(\mathrm{mg} / \mathrm{Kg} /$ day) were noted when comparing non-obese patients to obese patients. Similar studies which have examined the VCN average weight based daily dose $(\mathrm{mg} / \mathrm{Kg} /$ day using TBW) needed to achieve a therapeutic VCN trough levels found that doses for morbidly obese and non-obese patients did not significantly differ [11]. These findings suggest the use of TBW in calculating VCN mg/Kg doses for non-obese and obese patients.

The use of TBW and not ABW or IBW should be used in calculating doses at least initially until VCN serum levels can be obtained to adjust doses based on the patient specific PK parameters. This approach is supported by the Guidelines for the Therapeutic Monitoring of Vancomycin in Adult Patients which was published in 2009 by the American Society of Health-System Pharmacists (ASHP), Infectious Diseases Society of American (IDSA), and Society of Infectious Diseases Pharmacists (SIDP) [18]. The guidelines recommend a maintenance dose of $15-20 \mathrm{mg} / \mathrm{Kg}$ (based on TBW) every 8-12 hours in patients without renal disease in effort to try to achieve an AUC:MIC ratio of $>400$ (assuming the $\mathrm{MIC}$ is $\leq 1 \mathrm{mg} / \mathrm{Kg}$ ) [18].
The clinical utility of maintaining the AUC:MIC $>400$ has been endorsed by the ASHP/IDSA/SIDP as the PD parameter that can best predict the efficacy and clinical success of VCN with the best correlation to bacterial killing [19]. Although monitoring serum trough levels are common practice, the utility of the serum trough levels serve as a surrogate marker of AUC [19]. In a study including patients with lower respiratory tract infections, the patients who achieved an AUC:MIC ratio $>400$ exhibited bacterial eradication after 10 days compared with $>30$ days in those patients who did not reach the AUC/MIC ratio goal [20]. Despite the recommendations in the above mentioned guidelines, the majority of clinicians are hesitant to use such high weight based doses, especially in obese patients who may require more than 4 grams of VCN daily to maintain the desired AUC: MIC, as some studies have shown an increased risk of possible nephrotoxicity with the use of larger doses of VCN [21]. The common dilemma encountered by clinicians is whether targeting an AUC: MIC of $>400$ outweighs the risk of possible nephrotoxicity.

Despite the recommendation to use $15-20 \mathrm{mg} / \mathrm{Kg} \mathrm{TBW}$, the guidelines caution that even using such doses and targeting a VCN serum trough concentration of $15-20 \mathrm{mg} / \mathrm{L}$ may not achieving an appropriate AUC:MIC goal. A recent study compared the success rate of achieving a VCN AUC:MIC of $>400$ by using a weight and $\mathrm{CrCl}$ based nomogram with patients who received $15-20 \mathrm{mg} / \mathrm{Kg} / \mathrm{dose}$ (frequency based on $\mathrm{CrCl}$ ) [22]. Doses up to $1250 \mathrm{mg}$ every 8 hours $(3750 \mathrm{mg}$ daily) were utilized in the nomogram group in order to achieve the goal AUC:MIC of $>400$. Patients who were dosed based on the nomogram were 2 times as likely to achieve the AUC:MIC goal and were 2 times less likely to experience a VCN serum trough concentration $<15 \mathrm{mg} / \mathrm{L}$. The nomogram group was also approximately 4 times less likely to experience a steady-state VCN trough concentration $<10 \mathrm{mg} / \mathrm{L}$ [22]. These results support the claim that dosing patients based on $15-20 \mathrm{mg} /$ $\mathrm{Kg}$ may not be adequate to achieve the PD goal needed for VCN efficacy. In a separate study which examined the association between VCN AUC:MIC and the mortality risk in patients with MRSA bacteremia and endocarditis, an AUC:MIC ratio of $<211$ was associated with a greater than four-fold increase in attributable mortality compared to patients with an AUC:MIC of $>211$ [23]. Of note, patients in the AUC:MIC of $<211$ had an average dose of $16 \mathrm{mg} / \mathrm{Kg} /$ day while patients in the AUC:MIC $>211$ group had an average VCN dose of $22 \mathrm{mg} / \mathrm{Kg} /$ day thus further supporting the need for aggressive dosing of VCN in all patients particularly obese patients who would have a significantly lower AUC compared to a non-obese patient if given the a lower mg/ $\mathrm{kg} /$ day $\mathrm{VCN}$ dose [23]

A recent study of $\mathrm{VCN}$ in obese patients aimed to compare the ability of two various VCN doses to attain target trough levels and the incidence of nephrotoxicity [24]. A new VCN dosing regimen developed specifically for obese patients was evaluated using a loading dose of 20 $25 \mathrm{mg} / \mathrm{kg}$ based on ABW, followed by maintenance doses of $10 \mathrm{mg} / \mathrm{kg}$ every 12 hours, or $15 \mathrm{mg} / \mathrm{kg}$ every 24 hours, compared to an original protocol of a loading dose followed by $15 \mathrm{mg} / \mathrm{kg}$ every $8-12$ hours based on ABW. Obese patients in the study were those weighing over $100 \mathrm{~kg}$, with TBW exceeding $140 \%$ of IBW. 74 patients were evaluated using the revised protocol, compared with 64 patients who received the original protocol. First target trough obtainment rates were $59 \%$ with the revised protocol, compared with $36 \%$ with the original protocol $(p=0.006)$. These findings suggest loading doses of $20-25 \mathrm{mg} / \mathrm{kg}$ are effective for utilization in an obese patient population. In addition, VCN dosed at $10 \mathrm{mg} / \mathrm{Kg}$ every 12 hours resulted in a subtherapeutic trough of $<10$ $\mathrm{mg} / \mathrm{L}$ in $23 \%$ of the patients while $15 \mathrm{mg} / \mathrm{Kg}$ given every $8-12$ hours resulted in $9 \%$ of the patients with a subtherapeutic VCN trough $<10$ 
$\mathrm{mg} / \mathrm{L}$. Of note, the latter group required an average of $34 \pm 7 \mathrm{mg} / \mathrm{Kg} /$ day and yet achieved an average VCN trough of $>15 \mathrm{mg} / \mathrm{L}$ in approximately $55 \%$ of the patients in the given group (compared to an average trough concentration of $>14 \mathrm{mg} / \mathrm{L}$ in approximately $18 \%$ of patients on VCN $10 \mathrm{mg} / \mathrm{Kg}$ every 12 hours). Low rates of nephrotoxicity occurred with both protocols (2.7\% for revised and 3.1\% for original, $p=1.00$ ). Based on such evidence, doses above the standard $15-20 \mathrm{mg} / \mathrm{Kg}$ are needed in the majority of obese patients to achieve both a therapeutic VCN trough concentration and an appropriate AUC:MIC ratio.

\section{Use of vancomycin loading dose}

Administering a VCN loading dose helps achieve a AUC:MIC ratio of at least 400 and a therapeutic VCN trough concentration within the initial 24 hours of therapy which can be correlated to improved clinical outcomes and accelerated bacterial eradication. This has been supported by a study that found that delayed treatment was found to be an independent and strong predictor for mortality in patients with MRSA bacteremia [25].

The effectiveness of utilizing loading doses based on TBW in obese patients has begun to show improved clinical outcomes as it ensures an earlier achievement of AUC/MIC ratio $>400 \mathrm{mcg} /(\mathrm{h} / \mathrm{mL})$ [19]. Based on previously discussed findings, loading doses of $>20 \mathrm{mg} / \mathrm{Kg}$ based on TBW are required to achieve a therapeutic VCN trough concentration and AUC:MIC prior to achieving steady-state levels [24]. An additional study aimed to determine the percentage of critical patients in an intensive care unit (ICU) on VCN dosed based on the MM-USCPACK software who obtained a VCN serum trough concentration of $\geq$ $15 \mathrm{mg} / \mathrm{L}$ [26]. In this study, both non-obese (69\%) and obese (31\%) patients were included. The average BMI of all included patients was $25.8 \mathrm{~kg} / \mathrm{m}^{2}$. VCN loading doses of $\geq 25 \mathrm{mg} / \mathrm{Kg}$ (mean loading dose of $28 \mathrm{mg} / \mathrm{Kg}$ TBW) was administered to 20 patients (37\%) and no loading dose to the remaining 33 patients (63\%). Results showed that $58 \%$ of patients failed to achieve a VCN trough concentration of $>15 \mathrm{mg} / \mathrm{L}$. In addition, obesity was shown to be an independent predictor of not achieving target trough concentration.

Despite some variability in VCN pharmacokinetics between obese and hemodialysis patients, these findings are further supported by studies using loading doses in hemodialysis patients. In one study, use of a $20 \mathrm{mg} / \mathrm{Kg}$ loading dose based on dry-body weight led to subtherapeutic levels in $50 \%$ of patients, thus highlighting that the use of $<25 \mathrm{mg} / \mathrm{Kg}$ and a weight other than TBW will result in a significant percentage of patients not reaching therapeutic goals [27]. Another study showed that $20 \mathrm{mg} / \mathrm{Kg}$ would result in a mean VCN peak level of 16.1-30.3 mg/L explaining how other studies have shown that $50 \%$ or less of patients reach a therapeutic trough concentration following a loading dose of 20 $\mathrm{mg} / \mathrm{Kg}$ [28]. Of note, the authors of the study concluded that univariate analysis revealed significant correlation between dose in milligrams per kilogram based on TBW and the resultant trough concentration in this population, and that TBW had a stronger correlation with trough level than did IBW or ABW. Based on these recent studies, growing evidence supports the use of $\geq 25 \mathrm{mg} / \mathrm{Kg}$ as a VCN loading dose to achieve more rapid therapeutic VCN concentration, followed by a maintenance doses of $\geq 20 \mathrm{mg} / \mathrm{Kg}$ to maintain therapeutic VCN trough concentrations for the duration of therapy $[29,30]$.

\section{Maximum dose of vancomycin in obesity}

The use of loading doses in the obese population will most likely require doses that exceed the typical maximum dose of $2 \mathrm{~g}$. In the obese population, patients have been noted to have received up to 6 grams of VCN per day in a study that evaluated a dosage regimen in this specific population [24]. Of note, these patients received a range of 4-6 grams per day with no observation of nephrotoxicity with a median length of therapy of 5.5 days. Although fewer prospective studies have been published regarding dosing strategies for the obese population, recommendations concerning weight-based dosing, loading doses, and AUC:MIC should be applied to patients across the spectrum of BMI classes to improve clinical outcomes and decrease treatment failures.

\section{Adverse events with vancomycin in obesity}

The most significant adverse effects attributed to VCN therapy are nephrotoxicity and red-man syndrome. Both adverse effects have significantly decreased in incidence over the past decade following improved fermentation methods and increased VCN purity from $70 \%$ to $95 \%[21,31,32]$. VCN nephrotoxicity has been attributed to renal tubular ischemia of the proximal tubule cells following oxidative effects of VCN [33]. Studies have shown that the incidence of VCN nephrotoxicity ranges from $<10 \%$ when administered alone and up to as high as $40 \%$ when combined with other nephrotoxic medications [34]. To the knowledge of the authors, only one study to date has assessed the incidence of VCN induced nephrotoxicity in obese patients. In this recently published study which was discussed previously, nephrotoxicity correlated with initial VCN trough concentrations of $>15 \mathrm{mg} / \mathrm{L}$, however, it was not observed in patients who received maintenance doses $>4$ g per day [24]. This finding may suggest that the safety profile for the use of VCN in doses of $4 \mathrm{~g}$ per day or greater may differ in obese patients compared to non-obese patients. Many clinicians are hesitant to use VCN doses larger than $4 \mathrm{gm}$ per day based on the results of a previous study which had shown that VCN in doses $>4$ g per day were associated with an increased incidence of nephrotoxicity (hazard ratio (HR) of $4.37(2.96-6.45, p<0.001)$ especially in obese patients as the same study showed that a weight of $\geq 101.4 \mathrm{Kg}$ was also associated with increased risk of nephrotoxicity (HR 3.44, range of 2.40-4.93, $p=0.001$ ) [21]. One suggested approach is to administer larger doses of vancomycin at a maximum of $\mathrm{mg} / \mathrm{h}$ thus providing the patient with the optimal dose while avoiding a toxic peak concentration [30]. More recent studies aimed to specifically to address the efficacy and adverse events of $\mathrm{VCN}$ in obese patient will hopefully guide the future approach to how VCN should be dosed and monitored in this population.

\section{Conclusion}

More VCN PK and PD studies need to be conducted in obese patients as the incidence of obesity is at an all-time high. These studies will help guide clinicians especially physicians and pharmacists as to determine the most appropriate doses and PD goals in their obese population. The majority of current studies focus on non-obese patients which provide us with a good starting point and guide as to what need to be discovered about the variability in $\mathrm{PK}$ and $\mathrm{PD}$ in obese patients compared to their non-obese counterparts. At this time, it prudent that clinicians apply the PD goals which have been studied in mixed or nonobese population to the obese population keeping in mind that obese patients will likely require both larger loading and maintenance doses while receiving vancomycin more frequently. Fortunately, vancomycin induced nephrotoxicity is mostly reversible, and therefore clinicians should be aggressive with vancomycin therapy to ensure efficacy until VCN serum concentrations can be obtained from the patients and appropriate dosage adjustments can be made.

\section{References}

1. Centers for Disease Control and Prevention. Overwieght and Obesity. 
Citation: Grace E, Goodbar NH, Foushee JA (2012) Optimizing Vancomycin Dosing in Obese and Morbidly Obese Patients with MRSA Infections. Adv Pharmacoepidem Drug Safety S1:003. doi:10.4172/2167-1052.S1-003

2. Centers for Disease Control and Prevention. MRSA Infections.

3. Blouin RA, Bauer LA, Miller DD, Record KE, Griffen WO Jr (1982) Vancomycin pharmacokinetics in normal and morbidly obese subjects. Antimicrob Agents Chemother 21: $575-580$.

4. Vance-Bryan K, Guay DR, Gilliland SS, Rodvold KA, Rotschafer JC (1993) Effects of obesity on vancomycin pharmacokinetic parameters as determined by using a Bayesian forecasting technique. Antimicrob Agents Chemother 37: 436-440.

5. Butterfield JM, Patel N, Pai MP, Rosano TG, Drusano GL, et al. (2011) Refining vancomycin protein binding estimates: Identification of clinical factors that influence protein binding. Antimicrob Agents Chemother 55: 4277-4282.

6. Winter ME (1994) Vancomycin Pharmacokinetics. Basic Clinical Pharmacokinetics. $3^{\text {rd }}$ ed. Philadelphia, PA: Lippincott Williams \& Wilkins 474-499.

7. Bauer LA, Edwards WA, Dellinger EP, Simonowitz DA (1983) Influence of weight on aminoglycoside pharmacokinetics in normal weight and morbidly obese patients. Eur J Clin Pharmacol 24: 643-647.

8. Krogstad DJ, Moellering RC, Greenblatt DJ (1980) Single-dose kinetics of intravenous vancomycin. J Clin Pharmacol 20: 197-201.

9. Moellering RC Jr, Krogstad DJ, Greenblatt DJ (1981) Vancomycin therapy in patients with impaired renal function: a nomogram for dosage. Ann Intern Med 94: 343-346

10. Thomson AH, Staaz CCE, Tobin CM, Gall M, Lovering AM (2009) Development and evaluation of vancomycin dosage guidelines designed to achieve new target concentrations. J Antimicrob Chemother 63:1050-1057.

11. Bauer LA, Black DJ, Lill JS (1998) Vancomycin dosing in morbidly obese patients. Eur J Clin Pharmacol 54: 621-625.

12. Demirovic JA, Pai AB, Pai MP (2009) Estimation of creatinine clearance in morbidly obese patients. Am J Health Syst Pharm 66: 642-648.

13. Murphy JE, Gillespie DE, Bateman CV (2006) Predictability of vancomycin trough concentrations using seven approaches for estimating pharmacokinetic parameters. Am J Health Syst Pharm 63: 2365-2370.

14. Cockcroft DW, Gault MH (1976) Prediction of creatinine clearance from serum creatinine. Nephron 16: 31-41.

15. Blouin RA, Kolpek JH, Mann HJ (1987) Influence of obesity on drug disposition. Clin Pharm 6: 706-714.

16. Morita K, Yamaji A (1995) Changes in the serum protein binding of vancomycin in patients with methicillin resistant staphylococcus aureus infection: The role of serum alpha-1-acid glycoprotein levels. Ther Drug Monit 17:107-112.

17. Grace E (2012) Altered vancomycin pharmacokinetics in obese and morbidly obese patients: What we have learned over the past 30 years. J Antimicrob Chemother 67:1305-1310.

18. Rybak M, Lomaestro B,, Rotschafer JC, Moellering R, Craig W, et al. (2009) Therapeutic monitoring of vancomycin in adult patients: A consensus review of the American Society of health system Pharmacists, the Infectious Disease Society of America, and the Society of Infectious Disease Pharmacists. Am J Health Syst Pharm 66: 82-98.

19. DeRyke CA, Alexander DP (2010) Optimizing vancomycin dosing through pharmacodynamic assessment targeting area under the concentration-time curve/minimum inhibitory concentration. Hosp Pharm 44: 751-765.

20. Moise-Broder PA, Forrest A, Brimingham MC, Schentag JJ (2004) Pharmacodynamics of vancomycin and other antimicrobials in patients with Staphylococcus aureus lower respiratory tract infections. Clin Pharmacokinet 43: 925-942.

21. Lodise TP, Lomaestro B, Graves J, Drusano GL (2008) Larger vancomycin dose (at least 4 grams per day) are associated with an increase incidence of nephrotoxicity. Antimicrob Agents Chemother 52:1330-1336.
22. Croes S, Neef C, Stolk LM (2011) Comment: Outcomes associated with $\mathrm{AUC}_{24} / \mathrm{MIC}$ nomogram dosing of vancomycin. Ann Pharmacother 45: 1313 1314.

23. Brown J, Brown K, Forrest A (2012) Vancomycin AUC24/MIC ratio in patients with complicated bacteremia and infective endocarditis due to methicillin resistant staphylococcus aureus and its Outcomes associated with attributable mortality during hospitalization. Antimicrob Agents Chemother 56: 634-638.

24. Reynolds DC, Waite LH, Alexander DP, DeRyke CA (2012) Performance of vancomycin dosage regimen developed for obese patients. Am J Health Syst Pharm 69: 944-950.

25. Lodise TP, McKinnon PS (2005) Clinical and economic impact of methicillin resistance in patients with Staphylococcus aureus bacteremia. Diagn Microbio Infect Dis 52:113-122.

26. Aubron C, Corallo CE, Nunn MO, Dooley MJ, Cheng AC (2011) Evaluation of the accuracy of a pharmacokinetic dosing program in predicting serum vancomycin concentrations in critically ill patients. Ann Pharmacother 45:1193 1198

27. Vandecasteele SJ, De Bacquer D, De Vriese AS (2011) Implementation of dose calculator for vancomycin to achieve target trough level of $15-20 \mu \mathrm{g} / \mathrm{ml}$ in persons undergoing hemodialysis. Clin Infect Dis 53:124-129.

28. Brown M, Polisetty R, Gracely EJ, Cuhaci B, Schlednt HP (2011) Weight based loading of vancomycin in patients on hemodialysis. Clin Infect Dis 53: 164-166.

29. Li J, Udy AA, Kirkpatrick CM, Lipman J, Roberts JA (2012) Improving vancomycin prescribing in critical illness through a drug use evaluation process: A weight-based dosing intervention study. Int J Antimicrob Agents 39: 69-72.

30. Wang JT, Fang CT, Chen YC, Chang SC (2001) Necessity of loading dose when using vancomycin in critically ill patients. J Antimicrob Chemother 47 246

31. Bailie GR, Neal D (1988) Vancomycin ototoxicity and nephrotoxicity, a review. Med Toxicol 3: 376-386.

32. Lodise TP, Patel N, Lomaestro BM, Rodvold KA Drusano GL (2009) Relationship between initial vancomycin concentration -time profile and nephrotoxicity Clin Infect Dis 49: 507-514

33. Fanos V, Cataldi L (2001) Renal transport of antibiotics and nephrotoxicity; a review. J Chemother 13: 461-472.

34. Farber BF, Moellering RC Jr (1983) Retrospective study of the toxicity of preparation of vancomycin from 1974 to 1981 . Antimicrob Agents Chemother 23:138-141. 